\title{
Research on the Optimal Disposal of Space Debris for Private Firms Cost Analysis and Risk Aversion
}

\author{
Guangjin SHEN \\ Department of Electrical Engineering and Automation, North China Electric Power University, \\ Baoding, 071000, China
}

email: 18331136782@163.com

Keywords: Space Debris, Private Firms, Processing method, Cost Analysis, Risk Aversion

\begin{abstract}
Space debris, dispersed in space densely, has become a hot global issue since collisions happened increasingly. How to develop a time-dependent model to remove debris and benefit from this project is a rare commercial opportunity for private firms. Firstly, cost analysis and planning is one of the most important components. It clearly shows the costs in different process. Also, relied on opportunity cost principle, accountant principle, science of financial management and analytical hierarchy process, the risks and benefits are analyzed in detail. It turns out the methods are stable. It can help private firms clean up space debris and enjoy profits.
\end{abstract}

\section{Introduction}

Space debris, also called orbital debris, has become a hot global issue since collisions in space happened increasingly. According to data reported by NASA, there are more than 50,000 pieces of space debris around the Earth[1].

The quantity of space debris around the earth reached the tipping point already in 2011. And risks of being struck increase rapidly threatening safety of artificial satellites [2]. To reduce the risk of collision between spacecraft and orbit debris, the U.S. Space Surveillance Network tracks all debris larger than 10 centimeters [3].

Nowadays, scientists have proposed a large number of methods to remove the debris. Nevertheless, it is still difficult for us to solve this problem. Despite all this, more and more private firms long for a model to assess the different solutions to space debris problems so that they can benefit from this commercial opportunity.

\section{Cost Analysis and Planning}

At first, we take the first part of the whole problem, rocket launch costs, into consideration. And the stick figure of multistage rocket is shown.

In our model, the launch cost that is concerned with the stage of rocket and tonnage of fuels can be worked out. Through this model, we can get optimal solution. According to basic physical principles[4], the derivational.

Due to our assumptions, the rocket is attracted by the Earth only. The running velocity of devices can be worked out by Newton's law of universal gravitation.

In the rocket ascent, the rocket structure mass and fuel mass decrease simultaneously in a proportion of $\lambda$ and $1-\lambda$.

According to the conservation laws of momentum and energy, we get an equation.

$$
m(t) v(t)=m(t+\Delta t)-(1-\lambda) \frac{d m}{d t}(v(t)-u) \Delta t
$$

Then, we plug into Equ.1[5], another equation can be got. 


$$
v_{2}=3 \ln \left[\frac{\left(\frac{m_{1}}{m_{2}+m_{p}}+1\right)\left(\frac{m_{2}}{m_{p}}+1\right)}{\left(\frac{0.1 m_{1}}{m_{2}+m_{p}}+1\right)\left(\frac{0.1 m_{2}}{m_{p}}+1\right)}\right]=6 \ln \left(\frac{k+1}{0.1 k+1}\right)
$$

In the same way, we can calculate v3 and v4.

$$
\begin{aligned}
& v_{3}=9 \ln \left(\frac{k+1}{0.1 k+1}\right) \\
& v_{4}=12 \ln \left(\frac{k+1}{0.1 k+1}\right)
\end{aligned}
$$

When the final velocity of each stage rocket is equal to $v_{0}$, we achieve the minimum. Plug the final velocity, we get the results.

So, the cost is

$$
\left\{\begin{array}{l}
S_{2}=47.2 m_{p} \\
S_{3}=25 m_{p} \\
S_{4}=19 m_{p}
\end{array}\right.
$$

$$
\left\{\begin{array}{l}
C_{2}=c_{1}+c_{2}+47.2 m_{p} \\
C_{3}=c_{1}+c_{2}+c_{3}+25 m_{p} \\
C_{4}=c_{1}+c_{2}+c_{3}+c_{4}+19 m_{p}
\end{array}\right.
$$

According to assumptions, it's known that devises orbit the earth to do uniform circular motion. Then the motion state equation of devices can be listed based on law of uniform circular motion.

$$
\left\{\begin{array}{l}
\frac{G M m}{r^{2}}=m \frac{v^{2}}{r} \\
T_{1}=\frac{2 \pi r}{v}
\end{array}\right.
$$

At near surface, we can get the equation.

$$
\left\{\begin{array}{l}
\frac{G M m}{R^{2}}=m g \\
T_{1}=\frac{2 \pi r}{v}
\end{array}\right.
$$

Then, we get the equation as follow

$$
T_{1}=2 \pi \sqrt{\frac{(r-d)^{3}}{g R^{2}}}
$$

In the whole process, we can know the equation.

$$
\theta=2 \pi t\left(\frac{1}{T_{2}}-\frac{1}{T_{1}}\right)
$$

Then we plug T1 and T2 into Eq.9, the equation can be listed.

$$
t_{0}=\frac{\theta}{R\left(\sqrt{\frac{g}{(r-d)^{3}}}-\sqrt{\frac{g}{r^{3}}}\right)}
$$

It is easy to know that $\operatorname{Sum}=\beta \frac{T}{t}$, so the equation of Sum can be calculated. 


$$
\operatorname{Sum}=\beta \frac{T R}{\theta}\left(\sqrt{\frac{g}{(r-d)^{3}}}-\sqrt{\frac{g}{r^{3}}}\right)
$$

\section{Risk Aversion and Benefit Analysis:}

In the process of collision, we set up Three-dimensional modeling to predict potential risks. At first, the covariance matrix can be constructed due to errors in velocity and position of $X, Y$ and $Z$ axis[6]. On the basis of assumption, equations can be listed[6].

$$
\begin{gathered}
p(\overline{\tilde{\rho}})=\frac{1}{(2 \pi)^{3 / 2}(\operatorname{det} A)^{1 / 2}} \exp (-P) \\
P=\left(\overline{\tilde{\rho}}-\overline{\tilde{\rho}}_{0}\right)^{T} A^{-1}\left(\overline{\tilde{\rho}}-\overline{\tilde{\rho}}_{0}\right) / 2
\end{gathered}
$$

After a series of coordinate transformation, $A$ can be simplified to positively definite matrix.

$$
A=\left(\begin{array}{ccc}
\sigma_{1}^{2} & 0 & 0 \\
0 & \sigma_{2}^{2} & 0 \\
0 & 0 & \sigma_{3}^{2}
\end{array}\right)
$$

And its quadratic form Eq.12 means a surface of an ellipsoid whose three axes are $\sigma_{1}, \sigma_{2}$ and $\sigma_{3}$. We called this error ellipsoid representing the probability density distribution in collision

Through coordinates transform, extents of error to devices and space debris in their coordinates are projected to coordinates system for terrestrial equator inertia. At the same time, rendezvous reference systems will be built up every time they collide. Under this coordinate system, probability density function of devices and space debris positions errors can be got.

$$
\begin{aligned}
& f_{x . y}(x, y)=\frac{1}{2 \pi \sigma_{x} \sigma_{y}} \exp \left\{-\frac{1}{2}\left[\frac{\left(x-x_{0}\right)^{2}}{\sigma_{x}^{2}}+\frac{\left(y-y_{0}\right)^{2}}{\sigma_{y}^{2}}\right]\right\} \\
& x_{0}=R_{r e l} \sin \phi \\
& y_{0}=R_{r e l} \cos \phi
\end{aligned}
$$

In Eq.13, $\sigma_{x, y}$ is the standard deviation of devices (x) and space debris (y) positions.

$x_{0}$ and $y_{0}$ show the relative positions relationship of devices and space debris. Rrel is the size of projection that relative position vector projected to rendezvous plane. $\phi$, a relative vector, is the angle between the $y$-axis and the projection on rendezvous plane. In calculation, the device is regarded as a circle with a radius of $R_{S}$ located at the origin of coordinates. So, collision probability is equal to the integral of probability density in a certain safety zone.

- Yellow warning value: $P_{c} \leq 10^{-5}$. It's safe of devices in this rendezvous.

- Orange warning value: $10^{-5} \leq P_{c} \leq 10^{-4}$. It's slightly dangerous of devices in this rendezvous. In case that has no influence on devices' main tasks and doesn't damage the loan of devices, they should ignite to swing and avoid.

Red warning value: $P_{c} \geq 10^{-4}$. It's very dangerous of devices in this rendezvous, so they should ignite to swing and avoid immediately.

For private firm, only taking investment costs, returns and relevant risk into considerations to find a suitable sustainable method can clean up space debris, and in the meanwhile benefit [8]. Hence, the assessment of revenue risks for private firms is extremely significant if they want to clean up space debris.

According to Launch Cost Model we build, it is known that total mass can be determined once quantity and variety of devices are determined. By comparing with different launch costs of different stage rocket, the minimum launch cost can be determined. That is, $C_{1}$ means minimum total launch cost and we can get the equation. 


$$
C=C_{1}+C_{2}+\text { mne }
$$

Then we consider the benefit problem. In assumptions, space debris distribute uniformly, so it can be predicted that space debris around devices also distribute uniformly. In this way, we can get following equation.

$$
P_{g}=\lambda r P_{0} D+\alpha t \eta+S_{2} \cdot n e+S u m \cdot S_{1} \cdot n e
$$

And we know equations about $B$, ne and Sum (Eq.13).

$$
\begin{gathered}
B=P_{g}-C \\
n e=v_{0} t
\end{gathered}
$$

Combining Eq14, Eq.15, Eq.16 and Eq.17, we can get the equation.

$$
B=\lambda r P_{0} D+\alpha t \eta+v_{0} t S_{2}+S_{1} v_{0} t \cdot \operatorname{Sum}-\left(C_{1}+C_{2}+m v_{0} t\right)
$$

- When $\alpha \eta+\left(S_{2}+S_{1} \cdot S u m-m\right) v_{0}>0$, the slope of figure is positive, and private firms can make a profit.

- When $t=\frac{2\left(C_{l}+C_{c}-\lambda r P_{0} D\right)}{v_{0}\left(S_{2}+S_{1} \cdot \text { Sum }-m\right)+\alpha \eta}$, private firms will pay back their investment.

\section{Results and Analysis}

The Impact of Error Matrix A. The relative size of error matrix is limited from zero to five. Changing the component of devices' velocity in rendezvous (L), we draw a curve about the relationship of Pc and A when $\mathrm{L}=500,700,900$.

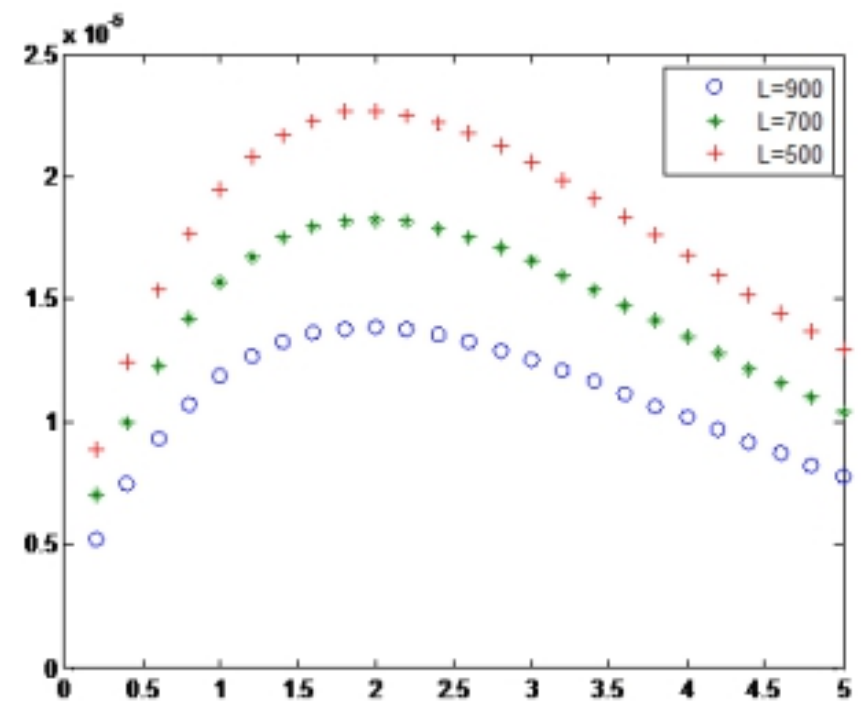

Fig.1 The curve about the relationship of $P_{C}$ and $A$

From Fig.1, when error value $\mathrm{A}$ is very small, the value of $P_{c}$ is also very small. When error value increasing, and so do PC increase. And when error value increases to an extent, $P_{c}$ is going to decrease. That is, $P_{c}$ is increasing rapidly when it gets to the maximum with the change of error value, so it is important of this curve on early warning. All in all, results are not sensitive to different values of A in a large range[9].

The Impact of $R_{s}$. In different values of $R_{s}$, we draw the following figure. Each curve represents the variation of $P_{c}$ with the change of error matrix in different values of $R_{s}$ [10].

AS Fig. 2 shows, when $R_{s}$ increasing, $P_{c}$ is also increasing, but it means that collision warning conservative. In conclusion, RS has little influence on $P_{c}$ and we don't need to set size of the safety radius, namely, RS has little influence on collision. 


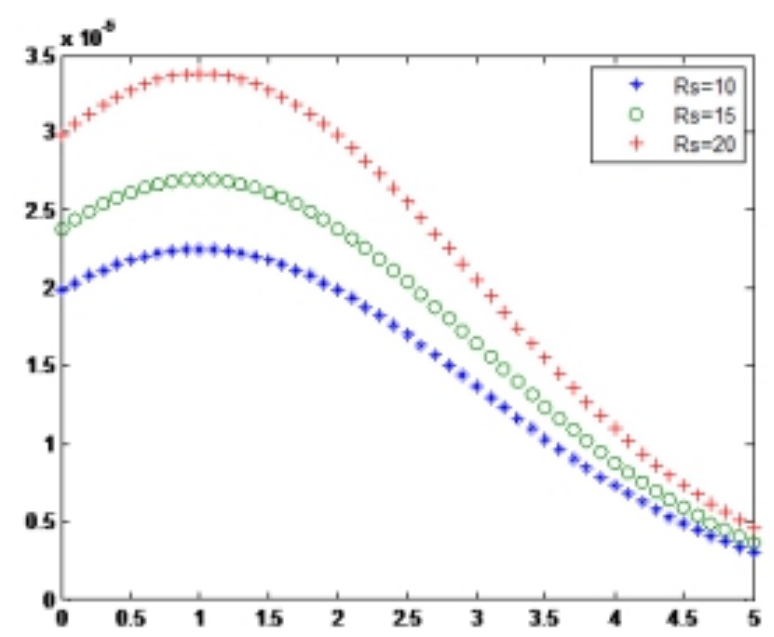

Fig.2 The curve about the relationship of $P_{C}$ and $R_{S}$

\section{Conclusion}

What we have discussed above that can clean up space debris and make profits for private firms includes launch costs, handling methods of different sizes of space debris, assessment of revenue risks for private firms and comparison of different options. Taking these actual factors into consideration, we developed four typical models_-Launch Cost Model, Cleaning Model, Collision Model and Revenue Risk Assessment Model to help private firms choose an optimal scheme. In addition, we make a comparison with three cleaning methods, that is, laser removing, satellite cleaning and suicide satellite eliminating. Through fuzzy evaluation method, we draw a conclusion that laser removing is more efficient in real life. All in all, it is recommended for private company adopt the combination of eliminating and avoid debris whereas the actual conditions. It is well established that building a laser removing base and Collision warning system can be the most valuable approach. Private firms can enjoy profits of removing space debris through our models.

\section{References}

[1] Gene Stansbery NASA's Orbital Debris Program Office. NASA’s Orbital Debris Program Office Briefing to the NASA Advisory Council.

[2] http://www.nasa.gov/sites/default/files/files/OrbitalDebrisProgramOffice.pdf

[3] The Earth Surrounded By Man-made Space Junk.

[4] http://www.junqing360.com/jiemi/20416.html. 2015.

[5] NASA Orbital Debris Program Office. (2009, July). Orbital debris frequently asked questions. Accessed September 11, 2009.

[6] Zhu Chengzhi. Discussion the minimum launch costs of multistage rocket scheme. Qingdao University (Natural Science), 02:25-33,2012,

[7] Wang Ruopu. Researches on environment model of space debris. PLA Information Engineering University, 2010.

[8] Cheng Tao. Collision Probability Analysis and Application of Cataloged Space Debris. Graduate Institute School (Space Science and Applied Research Centre), 2006.

[9] Xu Xiaoli, Xiong Yongqing. Researches on probability calculation about nonlinear relative motion of space debris. Astronomical Journal, 01:73-85.11,2011.

[10] Molly K. Mancauley. The economics of space debris: Estimating the costs and benefits of debris mitigation. Acta Astronautica, 2015. 